PFC/JA-88-38

\title{
Effect of Local Shear on Drift Flurtuation \\ Driven Transport in Tokamaks
}

\author{
J. Kesner
}

April 1989

\begin{abstract}
Plasma Fusion Center
Massachusetts Institute of Technology

Cambridge, Massachusetts 02139 USA
\end{abstract}

Submitted for publication in: Nuclear Fusion 
Effect of Local Shear on Drift Fluctuation Driven Transport in Tokamaks

\author{
J. Kesner \\ Massachusetts Institute of Technology \\ Cambridge, Massachusetts
}

\begin{abstract}
A theory based electron thermal diffusivity has been proposed recently which is based on electron drift wave turbulence. When account is taken for the effect of local shear changes that are produced in high poloidal beta equilibrium this theory is seen to predict the observed improvement of tokamak confinement with plasma current. Confinement is seen to be good at low poloidal beta, degrade as poloidal beta increases, and then enter an improved transport region at high poloidal beta.
\end{abstract}




\section{Introduction}

A theoretical understanding of tokamak thermal transport has not been well established and as a result the community has depended on empirical formulas. One empirical form proposed by Goldston for strongly heated tokamaks [1] has the form

$$
\tau_{E}=0.037 \frac{I_{p} R^{1.75} \kappa^{1 / 2}}{a^{.37} P_{a u x}^{1 / 2}}
$$

with $I_{p}=$ plasma current in MA, $\kappa$ the ellipticity, $\mathrm{R}$ and a the major and minor radii in $\mathrm{m}$, and $P_{a u x}$ the auxiliary power in MW. It is evident from Eq. 1 that tokamak confinement improves with current and degrades with auxiliary power, a troublesome result. Only a first principle theory can give insight into the origin and implications of this result.

The electron thermal diffusivity that results from both electromagnetic and electrostatic drift wave turbulence in a collisionless plasma has been evaluated by Horton, Choi, Yushmanov and Parail [2]. They assume the presence of a fixed amplitude spectrum of electron temperature gradient driven driven drift waves, the so-called $\eta_{e}$ modes, and calculate the resulting particle transport. For the short wavelength electrostatic and electromagnetic waves, they choose a wave spectrum with $\rho_{e}<2 \pi / k_{\perp}<c / \omega_{p e} . \omega_{p e}$ is the electron plasma frequency and $\rho_{e}$ the electron gyroradius. Stochasticity results from the resonance between the trapped electron circulation frequency, $\Omega_{k}$, with the trapped electron bounce motion (for electromagnetic waves) or the wave difference frequency, $\Delta \omega[3]$. The circulation frequency is the ExB drift frequency with which a trapped electron circulates around the wave potential island structures (in $r-\theta$ space). The circulation frequency is only meaningful for trapped electrons. The untrapped electrons will jump in the poloidal plane an angle determined by the rotational transform with each transit around the torus and therefore cannot be trapped in the wave potential structure. Ref. [2] finds an electron thermal diffusivity that has the form:

$$
\chi_{e}=\epsilon^{1 / 2}\left(D_{e m} \frac{c^{2} \omega_{b e}}{\omega_{p e}^{2}}+D_{e s} \frac{\rho_{s} c T_{e}}{r_{n} e B}\right)
$$


where the $D_{e m}$ term comes from the short wavelength $\eta_{e}$ modes and the $D_{e s}$ term from longer wavelength drift waves. $\epsilon$ is the inverse aspect ratio, $\epsilon=a / R, \omega_{b \epsilon}=\sqrt{\epsilon} v_{\text {the }} / R_{0} q$ the electron bounce frequency, $v_{\text {the }}$, the thermal speed, and $q$ is the safety factor, $\rho_{s}$ the ion gyroradius at the electron temperature, $T_{e}$ the electron temperature and $r_{n}$ the density gradient scale length. $D_{e m}$ and $D_{e s}$ are adjustable constants of order unity for electromagnetic and electrostatic waves respectively that we will determine from a best fit to a data base. Eq. 2 expresses a strong degradation of confinement as $T_{e}$ rises. $\chi_{e}$ is shown to be independent of the wave amplitude over a wide range of amplitude.

$D_{e m}$ is shown to be strongly reduced by shear and although global and local shear are not differentiated in [2], the local shear on the outer torus is the relevant shear since only trapped particles can circulate on the wave potential islands. The effect of shear is to have the trapped electrons sample different potential islands as they drift in radius and thereby degrade the above mentioned resonance between $\Omega_{k}$ and $\omega_{b}$ or $\Delta \omega$.

We will extract the shear dependence shown by [2] from $D_{e m}$, i.e. $D_{e m} \rightarrow f_{l}(s) D_{e m}$, and consider that the relevant shear is the local shear $\left(s \rightarrow s_{l}\left(\epsilon \beta_{p}\right)\right)$. The local shear, $s_{l}$, is defined as the shear seen by the deeply trapped electrons; $s_{l}=\frac{r}{q} \frac{d q_{l}}{d r}(\theta=0)$. $q_{l}$ is the local safety factor in the region of the trapped particles, $q_{l}=\mathrm{B} \cdot \nabla \phi / \mathrm{B} \cdot \nabla \theta$ and $\phi$ and $\theta$ are the respective toroidal and poloidal angle variables. $\left(s_{l}\right.$ should more correctly by the bounce averaged local shear seen by trapped electrons but in order to observe trends most simply we have evaluates $s_{l}$ at the outermost point of the flux surface.) We furthermore postulate that a similar factor can be extracted from $D_{e s}$ for the longer wavelength drift modes.

We will show that equilibrium changes can have a marked effect on electron transport through the dependence on local shear. It is known that as poloidal beta increases the local shear on the outside of the torus decreases until at some critical beta,$\beta_{p} \approx 1$, the local shear reverses direction. Fig 1 shows contours of local shear for a modestly shaped plasma at $\epsilon \beta_{p}=0.7$. The local shear has reversed within the cross hatched region. Thereafter higher poloidal beta leads to more strongly reversed shear. The detailed behavior of local shear with poloidal beta depends on the plasma current density profile as well as 
on plasma shaping, but the reduction and reversal of local shear with increasing poloidal beta is always observed in calculations with reasonable (monotonically decreasing) current density profiles. For $\epsilon \beta_{p} \geq 1$ this effect is responsible for the so-called "second stability" phenomena which has been predicted to restabilize MHD ballooning modes [4]. We shall see presently that it is through the $\epsilon \beta_{p}$ driven equilibrium changes of the shear appearing in the drift wave transport theory that an $\epsilon \beta_{p}$, and therefore a current dependence enters $\chi_{e}$ and $\tau_{E_{e}}$.

Eq. 2 predicts an increase in thermal diffusivity with electron temperature and therefore with auxiliary heating at constant current. This degradation of confinement is accentuated by the reduction of local shear that accompanies the increase in poloidal beta. However we see that at high enough poloidal beta the local shear reverses and then increases (in the reverse direction) leading to a recovery of confinement.

Thus we expect two regions of good confinement at low and at high poloidal beta. Ohmic heated tokamaks always operate in the low poloidal beta region; more power requires more current and thereby reduces the poloidal beta. We would therefore not expect a degradation of confinement with ohmic power. On the other hand, experiments which heat at constant current may be expected to exhibit a sharp deterioration of confinement to accompany increases in poloidal beta when $\beta_{p} \lesssim 1$.

\section{Theoretical model of $\tau_{E e}$ and its dependence on $s_{l}$.}

A confinement time can be obtained from Eq. 2 by taking $\tau_{E}=a^{2} / 4 \chi$.

$$
\tau_{E e}=\frac{66}{f_{l}\left(\epsilon \beta_{p}\right) D_{e m}} \frac{n_{e} a R^{2}}{\sqrt{T_{e}}\left(1 / q+A \beta_{e}\right)}
$$

with

$$
A=\frac{1}{2} \frac{D_{e s}}{D_{e m}} \frac{1}{\epsilon^{3 / 2}} \sqrt{\frac{m_{i}}{m_{e}}} .
$$

The dependence on local shear, $f_{l}\left(s_{l}\right)$ is exhibited explicitly in Eq. 3 and $s_{l}=s_{l}\left(\epsilon \beta_{p}\right)$. The confinement thus derived ignores profile factors and is approximate. 
The dependence of the diffusion coefficient on magnetic shear is obtained numerically in Ref. [2] for electromagnetic modes. We fit the ref [2] result with an exponential form and replace global by local shear to obtain

$$
f_{l} \approx 0.65 \exp \left(-3\left|s_{l}\right|\right)+0.05
$$

To estimate the local shear dependence on poloidal beta we have performed equilibrium calculations using a version of the PEST code [5] that has been modified [6] to produce current profiles having low edge current density for a fixed edge safety factor, $q_{a}$. In the first series of calculations we fix $q_{0}=1, q_{a}=4, A=3$ for both a circular and $D$ shaped (elongation, $\kappa=1.5$, triangularity, $\delta=0.5$ ) plasma. The ellipticity and triangularity of the plasma boundary are defined by assuming the following form for the plasma boundary:

$$
\begin{aligned}
& Z(\theta)=\kappa r \sin (\theta-\delta \sin \theta), \\
& R(\theta)=R_{0}+\xi(r)+a \cos \theta .
\end{aligned}
$$

With $\xi(r)$ is the shift of the center of the elliptic flux surface, $\kappa$ parameterizes the ellipticity and $\delta$ the triangularity.

Fig. 2 shows the local shear vs. poloidal beta at the outer torus and at the flux surface that is halfway out in poloidal flux. A similar trend of reduced and reversed local shear is observed in both cases although the shear reduces sooner for the $D$-shaped equilibrium. The observed high beta shear reversal was observed to occur similarly for all reasonable current profiles.

In Fig. 3 we plot $\tau_{E e}$ vs $T_{e}$ at fixed current from Eq. 3 (solid line) for the $D$-shaped tokamak $\left(q(a)=4, \overline{n_{e}}=3 \times 10^{13} \mathrm{~cm}^{-3}, B_{t}=1 T, \epsilon=0.33, \kappa=1.5, \delta=0.5\right)$. We choose $D_{e s}=1.5$ and $D_{e m}=0.6$ since these values minimize the mean square divergence from experimental D-III data in the data base [7] discussed below. As electron temperature increases the confinement time is observed to decrease until we reach a poloidal beta value 
when the outer torus local shear disappears. Thereafter a further temperature rise leads to an approximately constant $\tau_{E e}$ as the increasing reversed shear offsets the $T_{e}$ driven degradation. If we had assumed a larger $q_{a}$ value (lower plasma current) the confinement would have been reduced in the low temperature $\left(T_{e} \lesssim 1.4 \mathrm{KeV}\right)$ region but improved at higher $T_{e}$.

Fig. 4 plots $\tau_{E e}$ vs. plasma current for fixed plasma pressure $(\beta=3 \%)$. Above 300 KA confinement increases approximately linearly with current due to the increasing local shear that accompanies decreasing $\beta_{p}$. Below $300 \mathrm{KA}$ however we are in the reversed shear region and confinement increases with decreasing current. In fact at the confinement time is equal at 200 and $600 \mathrm{KA}$. The region of minimum confinement corresponds to where the local shear goes through zero, and this generally corresponds to a region of MHD (ballooning) instability. Thus both a stability and a transport barrier separate the the low beta region, where confinement scales linearly with current from the high beta region where we would predict confinement would scale inversely with current.

In Fig. 5 we compare the scaling law gotten from Eq. 2 with the Kaye-Goldston data base of auxiliary heated tokamaks [7]. Because of the complicated shear dependence on plasma shaping we only consider data from the the circular cross section machines, Asdex, DITE, PDX, TFR and TFTR. The data base only includes data from gettered L-mode experiments with $P_{i n j} \geq 2 P_{O H}$. We assume that the electron loss channel is dominant (and therefore $\tau_{E} \approx 2 \tau_{E e}$ ). The data base contains the measured $\tau_{E}$ values, plasma current, total input power and geometric parameters.

To quantify the comparison we define $\nu^{2} \equiv\left(\tau_{E e}-\tau_{e x p}\right)^{2} / \tau_{E e}^{2}$. We find that $\nu$ attains a minimum value, $\nu=0.23$ by setting $D_{e s}=8.5, D_{e s} / D_{e m}=2.7$ in Eq. 3 . If we leave out the local shear dependence (i.e. set $f_{l}=$ constant) we would obtain $\nu=0.27$. For comparison the Goldston L-mode fit gives $\nu=.24$. Thus we see that in this data base the local shear makes an observable improvement on the fit to the measured values.

The agreement between theory and experiment is good, indicating that electron drift wave transport might indeed be the dominant loss channel. The lack of a stronger shear dependence is not surprising since the data base is confined to relatively low poloidal beta 
shots. Other physical processes, including profile effects and losses in the ion channel, undoubtedly account for some of the scatter.

We compared Eq. 3 with a D-III data base [7]. We did not carry out the appropriate equilibrium calculations for DIII-D but instead used the local shear from the $\kappa=1.5$ series of calculations discussed above. $\nu^{2}$ again was minimized for $D_{e s} / D_{e s}=2.7$ with $D_{e s}=1.5$.

\section{Discussion and Conclusions}

We have shown that a self consistent treatment of the global equilibrium with the local transport problem may be a key to understanding tokamak transport. Taking $\chi_{e}$ [2] for electron drift waves and postulating that the local shear factor modelled by Eq. 4 should be in front of $\chi_{e}$ leads to the conclusion that confinement can be improved or degraded via the $\epsilon \beta_{p}$ dependent local shear. We find that two regions of good confinement exist, at low and at high poloidal beta. Ohmic heated tokamaks always operate in the low poloidal beta region; more power requires more current and thereby reduces the poloidal beta. Thus an increase of $T_{e}$ is offset by a decrease in poloidal beta.

Since we have only discussed the electron channel the improvement or deterioration of this channel is only meaningful if the ion channel does not dominate transport. Furthermore our conclusions are only valid if the plasma is collisionless.

We have not attempted to match the radial dependence of $\chi_{e}$ with experimental data. To do this properly one must not only know the radial temperature and density dependences, but also the local shear. (The reduction of local shear will tend to make $\chi_{e}$ increase locally with radius.)

In strongly auxiliary heated discharges with $\epsilon \beta_{p} \leq 1$, we predict that adding power will decrease $\tau_{E e}$ and that adding current would normally increase $\tau_{E e}$. The increase of $\tau_{E e}$ with current results from a reduction of the outer torus poloidal beta driven shear degradation. In the opposite limit of high poloidal beta $\left(\epsilon \beta_{p} \geq 1\right)$, reducing plasma current would improve confinement. Additionally operating in the low current regime is desirable for the following reasons: a. High $\beta_{p}$ can eliminate MHD ballooning modes (i.e. 
second stability), b. Low current will reduce volt-second requirements and/or current drive requirements.

Since a strong degradation of confinement accompanies auxiliary heating in high current discharges a transport as well as an MHD barrier must be overcome for entrance into the second transport regime. Recent experiments on the Versator II tokamak[8] have shown, that high beta equilibrium $\left(\epsilon \beta_{p} \approx 1\right)$ can be achieved in low current discharges in which the thermal plasma pressure remains low and current drive is employed. Both the plasma current and pressure are carried by energetic passing electrons which would not take part in the above mentioned transport. This leads to the possibility that one can access the high confinement region by starting with a high poloidal beta discharge and increasing the current (using RF current drive techniques) in proper proportion to the pressure during the heating phase so as to remain in the high confinement regime.

In comparing with the data base for strongly auxiliary heated experiments (Fig 5 ) one must keep in mind that we are only calculating one loss process, turbulent transport in the electron channel. The good agreement shown implies that electron channel drift wave turbulence may indeed be the dominant loss process.

If we consider strongly auxiliary heated devices and therefore only keep the high beta term in the denominator of Eq. 3 we can then apply the power balance (Eq. 6) to show $\tau_{E e} \propto P_{a u x}^{-0.6}$. Thus we obtain a similar power degradation as observed by Kaye and Goldston [7]. The improvement of $\tau_{E}$ with current in these empirical fits can be compared with Fig. 4 where we observe an approximately linear improvement of $\tau_{E e}$ with current in the high current regime. 


\section{Acknowledgements}

I would like to thank Dr. S. Kaye (PPPL) for supplying the data base for auxiliary heated experiments. I would also like to thank P. Hakkarainen for use of his modified version of PEST and J. Freidberg, B. Lane, D. Sigmar of MIT and L. Woods (Oxford) for helpful and stimulating discussions. This work was performed under U.S. DOE contract DE-AC02-78ET-51013. 


\section{References:}

1 R.J. Goldston, Plasma Physics and Controlled Fusion 26, (1986), 87.

2 W. Horton, D.-I. Choi, P.N. Yushmanov and V.V. Parail, Plasma Physics and Controlled Fusion 29, (1987) 901.

3 W. Horton, Plasma Physics and Controlled Fusion 27, (1985) 937.

4 B. Coppi, G.B. Crew and J.J. Ramos, Comments Pl. Phys. and Cont. Fus., 6,(1981) 109.

5 R.C. Grimm, R.L. Dewar and J. Manickam, J. Comput. Phys. 4, (1983) 94.

6 S.P. Hakkarainen and J.P. Freidberg, Bull. A.P.S., 32, (1987) 1772.

7 S.M. Kaye and R.J Goldston, Nuclear Fusion 25, (1985) 65.

8 S.C. Luckhardt, K.I. Chen, S. Coda, J. Kesner, R. Kirkwood, B. Lane, M. Porkolab and J. Squire, Phys. Rev. Lett. 62,( 1989) 1508. 


\section{Figure Captions:}

FIG. 1. Contours of local shear for a modestly shaped plasma at $\beta_{p}=0.7, \beta=3 \%, q_{a}=3$. Cross hatched areas contain negative shear.

FIG. 2. Local shear at the outer torus at a flux surface halfway out in poloidal flux for a) A circular outer boundary and b) A $D$ shaped boundary having $\kappa=1.5$ and $\delta=0.5$. We fix $\epsilon=0.33, q_{0}=1$ and $q_{a}=4$.

FIG. 3. $\tau_{E e}$ vs $T_{e}$ at fixed current for drift wave scaling law. $q(a)=4, \overline{n_{e}}=3 \times$ $10^{13} \mathrm{~cm}^{-3}, B_{t}=1 T, \epsilon=0.33, \kappa=1.5, \delta=0.5$.

FIG. 4. $\tau_{E e}$ vs. plasma current for fixed plasma pressure $\beta=3 \%, \overline{n_{e}}=3 \times 10^{13} \mathrm{~cm}^{-3}, B_{t}=$ $1 T, \epsilon=0.33, \kappa=1.5, \delta=0.5$.

FIG. 5. Experimentally measured $\tau_{E}$ vs theoretical value for circular boundary tokamaks in the Kaye [7] data base. 


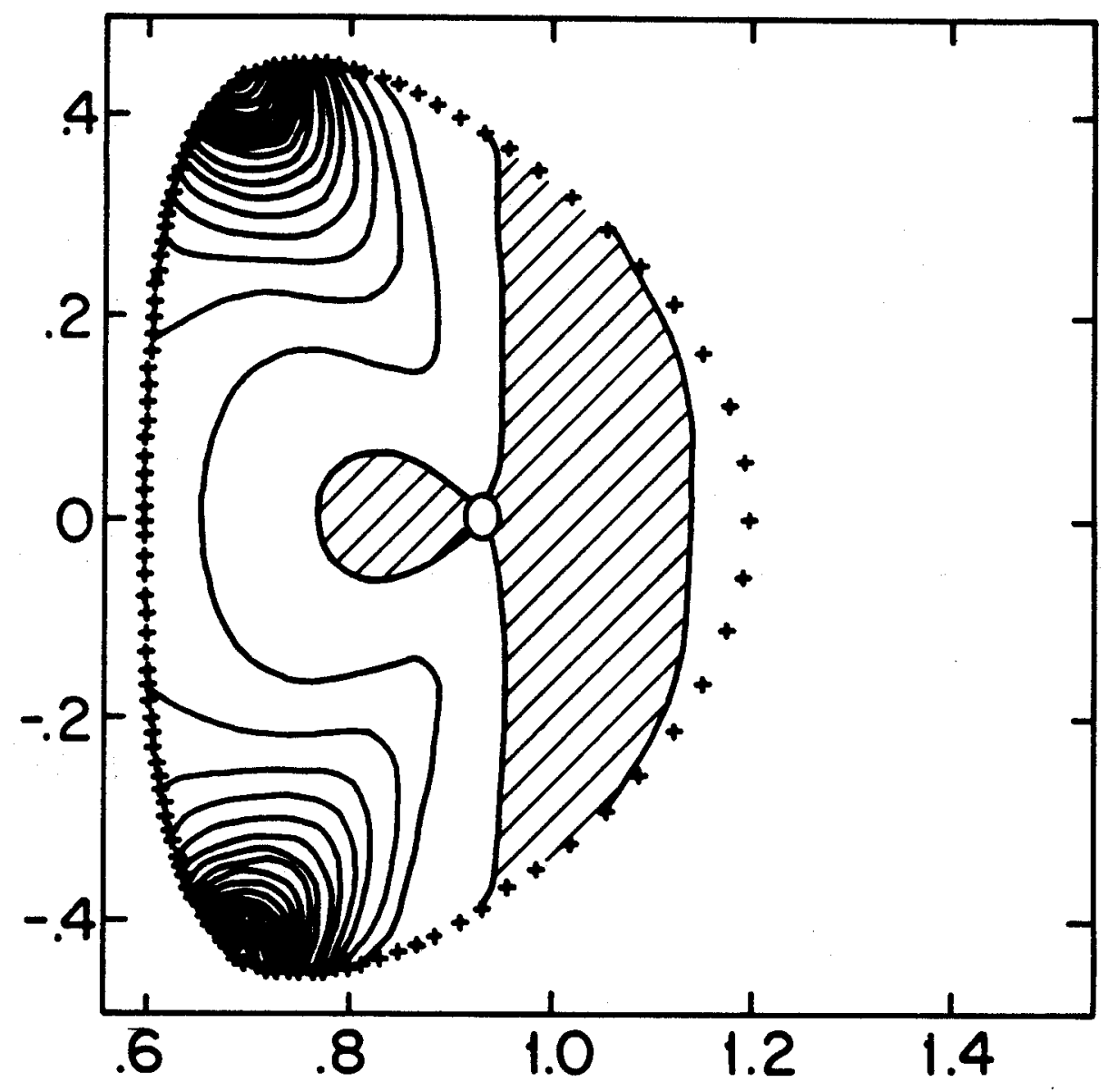



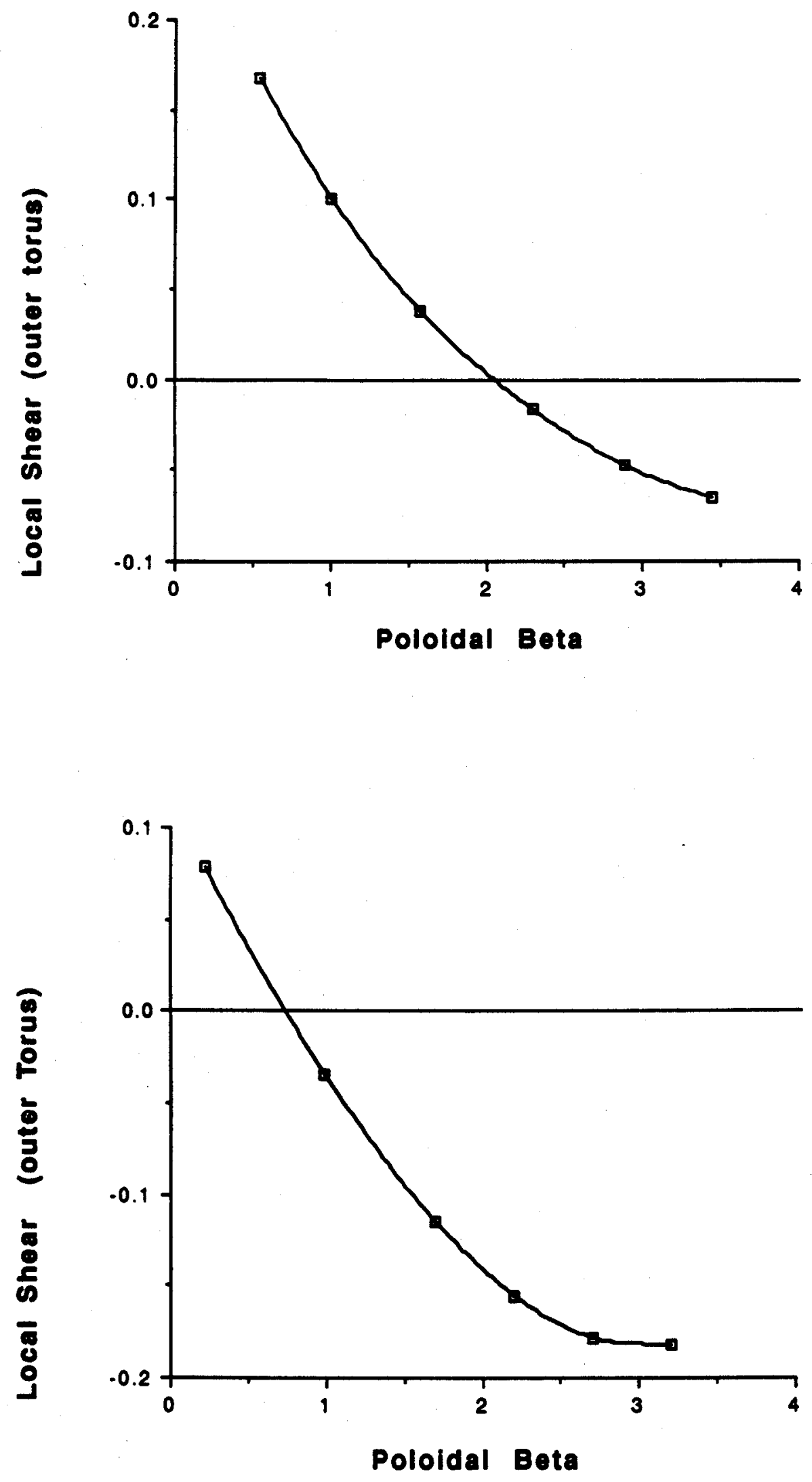


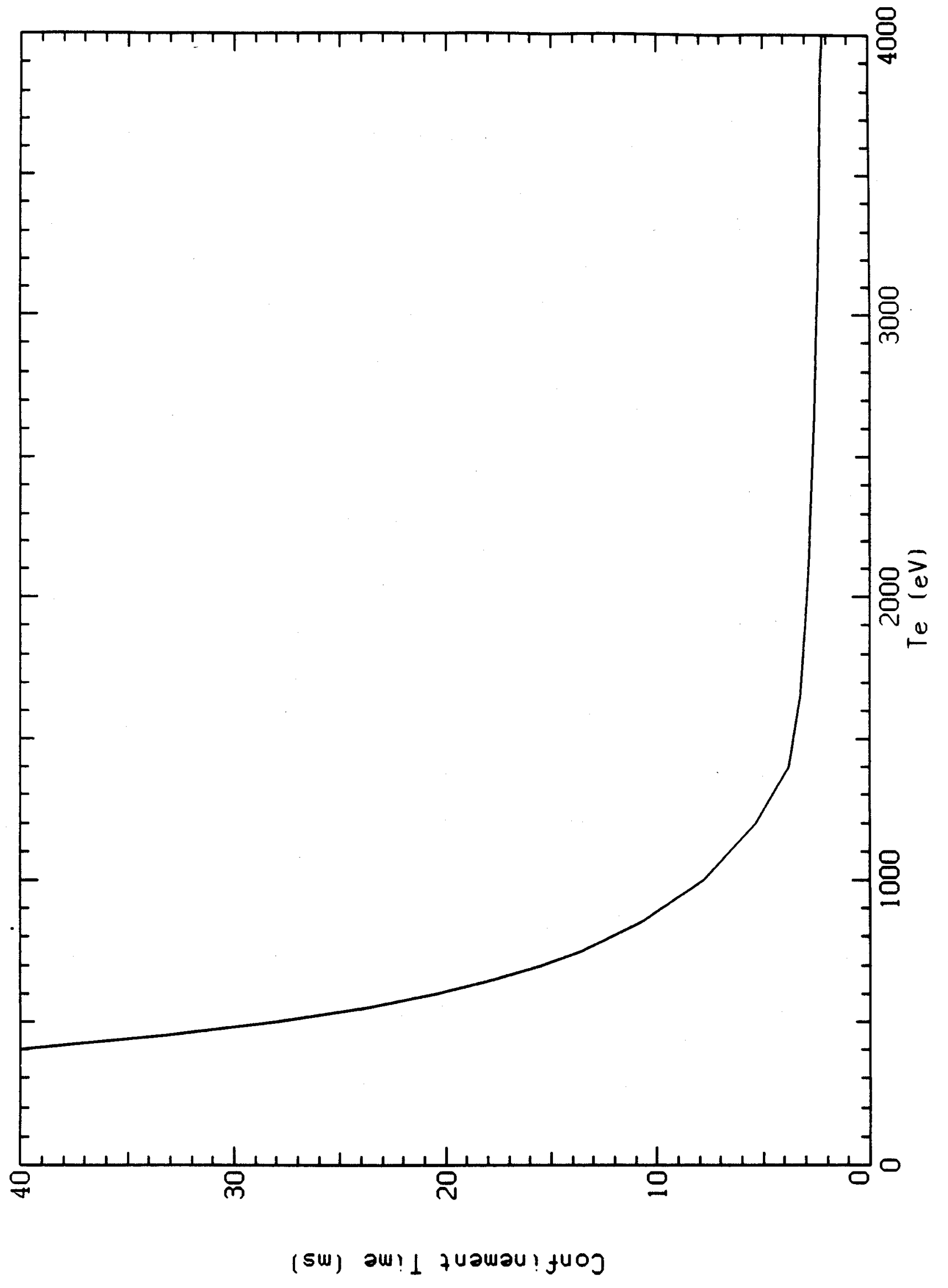




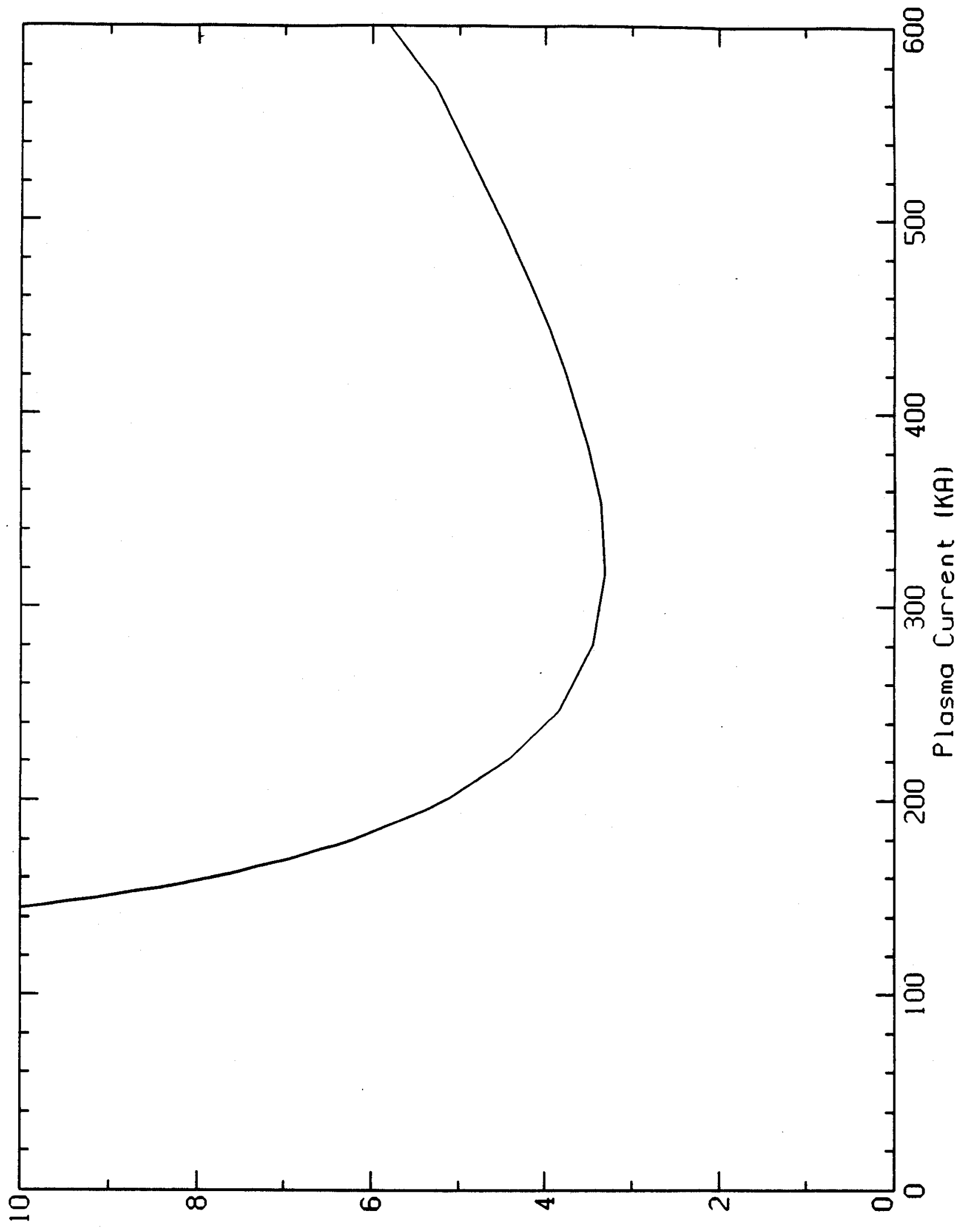

(sw) aw! q qawau! juoJ 


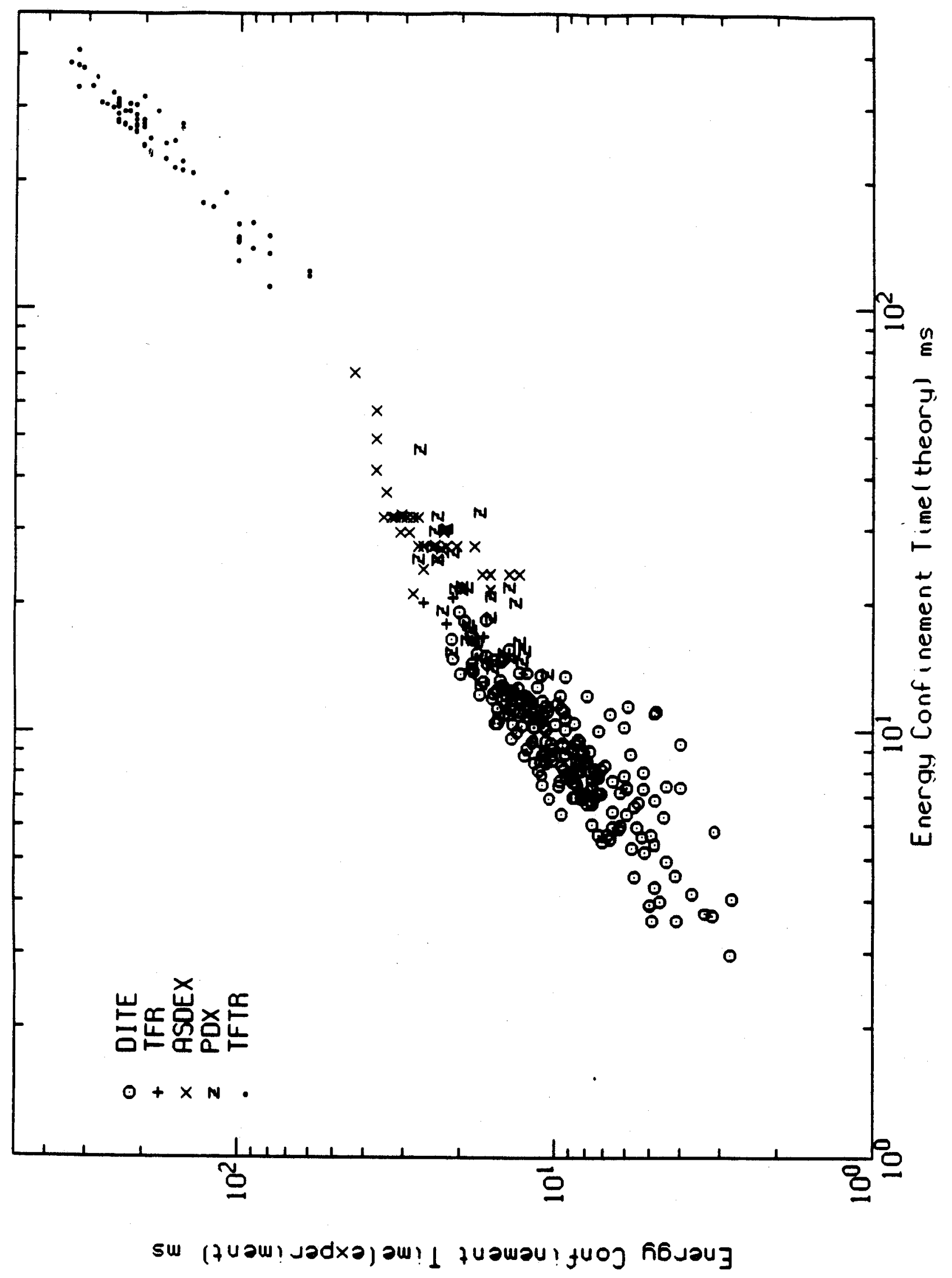

